\title{
Combinatoria del marcador discursivo pues y sus correspondencias en la lengua croata
}

\section{IVANA LONČAR}

Universidad de Zadar

Departamento de Estudios Iberorrománicos

Tfno. + 38523200572

E-mail: iloncar@unizd.hr

COMBINATORIA DEL MARCADOR DISCURSIVO PUES Y SUS CORRESPONDENCIAS EN LA LENGUA CROATA

RESUMEN: El marcador discursivo pues, uno de los marcadores con alta frecuencia de uso tanto en la comunicación escrita como oral, resulta ser poco definido, insuficientemente tratado y de dificil adquisición para los aprendices de español como lengua extranjera (Uribe Mallarino, 2006: 9). El presente trabajo versa sobre su combinatoria en el vasto ámbito de pragmalingüistica hispánica y los equivalentes traductológicos respectivos en la lengua croata, tanto desde el punto de vista de la lingüística contrastiva, como desde la perspectiva de la adquisición del español como lengua extranjera, con el fin de ofrecer un número considerable de correspondencias croatas partiendo de una investigación basada en los corpus lingüísticos.

PALABRAS ClAves: pues; marcador discursivo; combinatoria; corpus; español; croata.

SUMARIO: 1. Introducción. 2 Corpus de investigación. 2.1. Problemas específicos del corpus analizado. 3. Análisis traductológico. 4. A modo de conclusión.

\author{
JOSIPA MUŠURA \\ Universidad de Nebrija \\ Centro de Estudios Hispánicos \\ Tfno. + 34914521103 \\ E-mail: jmusura@nebrija.es
}

\section{COMBINATIONS OF DISCUR- SIVE MARKER PUES AND ITS EQUIVALENTS IN CROATIAN LANGUAGE}

ABSTRACT: The discursive marker pues, one of the markers with the highest frequency of use in both written and oral communication, it proves to be poorly defined and treated, just as its acquisition for learners of Spanish as a foreign language represents a big obstacle (Uribe Mallarino, 2006: 9). This paper deals with respective combinations in the vast field of Hispanic Pragmalingusitics, as well as with the translational equivalents in the Croatian language within the field of contrastive linguistics, and from the point of view of the acquisition of Spanish as foreign language, in order to offer a considerable number of Croatian correspondences based on a research within corpus linguistics.

KEY WORDS: pues; discursive marker; combinations; corpus; Spanish; Croatian.

SUMMARY: 1. Introduction. 2 . Investigation corpus. 2.1. Particular problems in the analysis of a corpus. 3. Translation analysis. 4. In conclusion.
COMBINATOIRE DU MARQUEUR DISCURSIF PUES ET SES EQUIVALENTS DANS LA LANGUE CROATE

RÉSUMÉ : Le marquer discursif pues, l'un des marqueurs à haute fréquence d'utilisation, tant dans la communication écrite que dans l'orale, s'avère mal défini, insuffisamment traité et d'une acquisition difficile pour les apprenants de l'espagnol comme langue étrangère (Uribe Mallarino 2006 : 9). Le présent travail traite le thème de sa combinatoire dans le vaste domaine de la pragmalinguistique hispanique et les équivalents traductologiques respectifs en croate dans le domaine de la linguistique contrastive, ainsi que du point de vue de l'acquisition de l'espagnol comme langue étrangère, afin d'offrir un nombre considérable de correspondances croates sur la base d'une investigation des corpus linguistiques.

MOTS CLÉS : pues; marquer de discours; combinatoire; corpus; espagnol; croate.

SOMMAIRE : 1 . Introduction. 2. Corpus d'investigation. 2.1. Problèmes spécifiques du corpus analysé. 3. Analyse traductologique. 4. En guise de conclusion.
Fecha de Recepción Fecha de Revisión Fecha de Aceptación Fecha de Publicación
$14 / 04 / 2016$

$17 / 08 / 2018$

$11 / 09 / 2018$

$01 / 12 / 2018$ 


\title{
Combinatoria del marcador discursivo pues y sus correspon- dencias en la lengua croata
}

\author{
IVANA LONČAR \& JOSIPA MUŠURA
}

\section{INTRODUCCIÓN}

En el vasto ámbito de pragmalingüística hispánica abundan en los últimos años los trabajos que desde varios enfoques analizan los marcadores discursivos como "partículas polifuncionales presentes en todos los registros del español contemporáneo" (Reyes, 1994, 2011; Escandell Vidal, 1996; Uribe Mallarino, 2006; Martí Sánchez, 2008, 2011, 2013; Briz Gómez 1993, 1994, 2001; Casado Velarde, 1991, 1993; Cueto Vallverdú, Domínguez García, 2006; Fuentes Rodríguez, 1993; Gili Gaya, 1943; La Rocca, 2011; López Bobo, 2003; Llopis-Cardona 2014; Martín Zorraquino, 1992, 1998, 1999; Moltolío, 1998; Portolés, 1998, 1999; Santana Marrero, 2014; Serrano, 1997). Se trata de un grupo heterogéneo de autores y sus respectivos trabajos de investigación, que en su totalidad engloban un número elevado de cuestiones teóricas y tipológicas relevantes de los marcadores discursivos. Precisamente por esta heterogeneidad, cabe destacar la diversidad de términos por los que se denominan dichas unidades (enlaces extraoracionales (Gili Gaya, 1943: 325-331), conectores (Martínez, 1997; Pons, 1998b), conectores argumentativos (Portolés 1989), conectores discursivos (Montolío, 1992), conectores pragmáticos (Briz 1993a, 1993b y 1994), conectores enunciativos (Lamíquiz 1994, 183-191), partículas discursivas (Martín Zorraquino 1992, Briz 1998), enlaces textuales (López García 1994: 107-116), operadores discursivos (Casado-Velarde, 1991 y 1993), ordenadores del discurso (Alcina y Blecua 1975, § 7.3.6), entre otros. Siguiendo la terminología propuesta por Martín Zorraquino y Portolés Lázaro (1999), optamos por el término marcadores del discurso y la clasificación elaborada por los autores.

El marcador del discurso pues es sin duda uno de los marcadores que presenta "una alta frecuencia tanto en la comunicación escrita como oral, y resulta ser poco definido, insuficientemente tratado y de dificil adquisición para los aprendices de español como lengua extranjera" (Uribe Mallarino, 2006: 9). El presente trabajo versa sobre su combinatoria en el vasto ámbito de pragmalingüística hispánica y los equivalentes traductológicos respectivos de estas unidades en la lengua croata. Tratándose de uno de los marcadores de mayor uso, consideramos que la presente investigación merece una particular mención en los estudios de pragmalingüística y a la vez aporta valiosos datos para los trabajos de lingüística contrastiva. El objetivo principal de nuestro trabajo no es proporcionar una panorámica de los estudios que tratan el tema del marcador discursivo pues y su combinatoria, sino más bien ofrecer un número considerable de equivalentes croatas a partir de una investigación basada en el corpus, siempre tomando en consideración la situación comunicativa. 
La lingüística croata, a diferencia de la española, carece de una investigación sistemática y compleja que verse exclusivamente sobre los marcadores discursivos (con excepción de Velčić, 1987). Más bien, solo encontramos a autores que, dentro de los campos de pragmática, sintaxis o fraseología, hacen mención a algún tipo de dichas unidades, hecho que complica aun más la comprensión y la reproducción de dichas unidades en croata.

\section{CoRPuS DE INVESTIGACIÓN}

La alta frecuencia del marcador discursivo pues fácilmente puede comprobarse en los corpus del español (CORPES: 99769 ocurrencias, CREA: 82 322, CORDE: $250583^{1}$. No obstante, se ha comprobado que la investigación y el análisis de la combinatoria del pues en los corpus lingüísticos manifiestan ciertas dificultades que detallaremos a continuación.

\begin{tabular}{|c|c|c|c|}
\hline $\begin{array}{c}\text { combinatoria } \\
\text { léxica }\end{array}$ & $\begin{array}{c}\text { ocurrencias (resultados } \\
\text { de la búsqueda) }\end{array}$ & $\begin{array}{c}\text { ocurrencias } \\
\text { descartadas }\end{array}$ & Total \\
\hline pues sí & 2450 & 1014 & 1436 \\
\hline pues bien & 2028 & 64 & 1964 \\
\hline así pues & 1558 & 20 & 2008 \\
\hline pues eso & 781 & 465 & 316 \\
\hline pues entonces & 411 & 0 & 411 \\
\hline pues nada & 376 & 154 & 222 \\
\hline pues mira & 245 & 56 & 189 \\
\hline pues la verdad & 126 & 85 & 41 \\
\hline pues mire & 117 & 20 & 97 \\
\hline pues vaya & 114 & 99 & 15 \\
\hline bueno pues & 80 & 8 & 2 \\
\hline pues ve & 73 & 71 & 48 \\
\hline sí pues & 51 & 3 & 47 \\
\hline pues fijate & 47 & 0 & 39 \\
\hline pues venga & 43 & 4 & 26 \\
\hline pues hombre & 26 & 0 & 24 \\
\hline pues fijese & 24 & 0 & 27 \\
\hline
\end{tabular}

Tabla 1: Las ocurrencias de la combinatoria del pues en CORPES XXI (consultado en marzo de 2016)

Partiendo de la explotación del Corpus del Español del siglo XXI que, como los demás corpus académicos, abarca todos los países de habla española, se ha confirmado que este produce el mayor número de ocurrencias relativas a las combinatorias de nuestro principal interés. De ahí que se manifiesten algunas variantes de dichas combinatorias, tales como pues mirá o pues fijate (formas verbales del voseo en imperativo, especialmente el de Argentina y Costa Rica).

\footnotetext{
1 Consultado en marzo de 2016.
} 


\subsection{PROBLEMAS ESPECÍFICOS DEL CORPUS ANALIZAdo}

Una vez realizada la búsqueda de ejemplos de uso del pues y sus respectivas combinatorias, a la hora de acotar el corpus de investigación se ha considerado imprescindible descartar las combinaciones denominadas en la Tabla 1. "ocurrencias descartadas". El primer grupo de ellas está formado por las combinaciones como: pues si/si, pues váyase, pues vete, pues mírate/miralo/mirelo/miré, dado el hecho de que el corpus como tal no reconoce ni los acentos gráficos ni las diferentes formas verbales, sea en pretérito indefinido (miré), sea en imperativo, con pronombres (mírate, míralo, etc.). El segundo grupo descartado consiste en aquellas ocurrencias que constituyen un elemento que no forma parte de la combinatoria del pues: por ejemplo, el adverbio de afirmación sí descartado de la unidad sí pues en algunos ejemplos no pertenece a la combinatoria del marcador, sino se trata de un sintagma adverbial en función de complemento directo ( $e l$ sî) o pronombres con preposición (para sî). La forma venga de algunas combinaciones de pues venga no es más que la $3^{a} \mathrm{p}$. sing. del imperativo (venir), igual que mira de pues mira $-2^{\text {a }}$ p. sing. del imperativo (mirar) - y vaya de pues vaya $-3^{\mathrm{a}} \mathrm{p}$. sing. del imperativo (ir), siendo este último también exclamativo (vaya manera). En cuanto al componente mire de la unidad pues mire/miré, puede ser tanto la $3^{\mathrm{a}} \mathrm{p}$. sing. del imperativo (mirar), $1^{\mathrm{a}} \mathrm{p}$. sing. del pretérito indefinido (mirar) como formar parte de una fórmula/locución verbal (mire Ud. por dónde). La forma ve de pues ve en algunos contextos corresponde a la $3^{a}$ p. sing. del imperativo (ir) o $3^{a}$ p. sing. del presente de indicativo (ver), hasta forma parte de algunas expresiones (vete tú a saber). El componente bueno del sintagma bueno pues a veces es un simple adjetivo, si de pues si (recordemos que el corpus no reconoce los acentos gráficos/ la tilde) una conjunción (oraciones condicionales), bien de pues bien un adverbio. Así de asi pues en algunos contextos no va unido al marcador, ni forma parte de su combinatoria, sino más bien se trata de un adverbio (pero no es así, pues la próxima semana tendremos otro). Lo mismo sucede con nada de pues nada que resulta ser un pronombre indefinido con función de sujeto (nada es irrelevante) o más bien un pronombre indefinido en función de complemento directo (nada podia decir). Con respecto a eso de la unidad pues eso, cabe decir que puede tratarse de un pronombre demostrativo con función de sujeto (eso no está bien). Por último, igual que en los ejemplos precedentes, la verdad de pues la verdad no siempre forma parte de la combinatoria, sino equivale a un sintagma nominal con función de sujeto (la verdad del hombre). Por lo tanto, a la hora de acotar el corpus de investigación, especialmente cuando se trata de combinaciones que se quieren presentar según la frecuencia de uso, resulta necesario analizar cada una de las ocurrencias con el fin de contar con datos fidedignos para la investigación. En la Tabla 2 . se presentan las combinatorias del pues, según la frecuencia de ocurrencias en el corpus analizado en el presente trabajo. 


\begin{tabular}{|c|c|}
\hline combinatoria léxica & número de ocurrencias \\
\hline así pues & 2008 \\
\hline pues bien & 1964 \\
\hline pues sí & 1436 \\
\hline pues entonces & 411 \\
\hline pues eso & 316 \\
\hline pues nada & 222 \\
\hline pues mira & 189 \\
\hline pues mire & 97 \\
\hline bueno pues & 72 \\
\hline sí pues & 48 \\
\hline pues fijate & 47 \\
\hline pues la verdad & 41 \\
\hline pues venga & 39 \\
\hline pues hombre & 26 \\
\hline pues fijese & 24 \\
\hline pues vaya & 15 \\
\hline pues ve & 2 \\
\hline
\end{tabular}

Tabla 2: Las combinatorias del pues más frecuentes en el CORPES XXI (consultado en marzo de 2016)

\section{ANÁlisis traductológico}

A lo largo de este apartado presentaremos los equivalentes traductológicos croatas de las combinatorias españolas del pues, con respectivas observaciones dentro del campo de lingüística contrastiva.

\begin{tabular}{|c|l|}
\hline Combinatoria léxica & \multicolumn{1}{|c|}{ Correspondencias en croata ${ }^{2}$} \\
\hline así pues & $\begin{array}{l}\text { tako da, znači, (a) to bi značilo, što bi značilo, iz toga slijedi, prema } \\
\text { tome, stoga, dakle }\end{array}$ \\
\hline pues bien & pa dobro, pa sad, dakle \\
\hline pues sí & pa da \\
\hline pues entonces & (e) pa vidiš, (e) pa onda, (e) pa da, (i) onda, a onda \\
\hline pues eso & pa (eto) to, pa tako, e tako, pa upravo to, pa upravo tako, baš tako \\
\hline pues nada & pa ništa, a ništa, ništa onda \\
\hline pues mira & $\begin{array}{l}\text { pa zamisli, ma zamisli, 'ajde zamisli/reci, daj samo zamisli, pa vidi, } \\
\text { a vidi, pa gle, a gle, dakle, ovako, (a) bože moj }\end{array}$ \\
\hline pues mire & pa vidite, pa gledajte, pa ovako, evo ovako \\
\hline bueno pues & dobro onda, pa dobro, dakle, daj/'ajde više, daj zbilja \\
\hline si pues & pa da, e da \\
\hline pues fijate & $\begin{array}{l}\text { e da, zamisli, a zamisli, ali zamisli, ma zamisli (ti to), možeš li vje- } \\
\text { rovati, a pazi }\end{array}$ \\
\hline pues la verdad & $\begin{array}{l}\text { pa zapravo, pa iskreno, pa najiskrenije, pa da ti pravo kažem, pa } \\
\text { da ti budem iskren, ako ćemo iskreno }\end{array}$ \\
\hline pues venga & 'ajmo, 'ajde, idemo, daj, daj 'ajmo, daj 'ajde, daj idemo \\
\hline pues hombre & pa, e, ali gle/vidi/daj, 'ajmo onda, 'ajde, da čujem \\
\hline pues fijese & a gledajte, ma zamislite, (e) pa nije tako, (e) pa neće ići/moći \\
\hline pues vaya & zamisli, ma zamisli, pa zamisli, tko bi rekao, ma da, gle čuda \\
\hline pues ve & pa gledajte, (a) pa gledaj \\
\hline Tabla 3: Correspara
\end{tabular}

Tabla 3: Correspondencias en croata de la combinatoria léxica del marcador pues

\footnotetext{
2 A diferencia del significado léxico de las correspondencias que se indica entre 'comillas simples', las traducciones literales de los equivalentes croatas se proporcionan entre corchetes, precedidas por un asterisco.
} 
Para empezar, cabe destacar que la traducción al croata de las siguientes combinatorias del pues no varía mucho a lo largo de los ejemplos del corpus analizado; incluso si dentro del discurso cambia la entonación, existe la posibilidad de intercambiar varias correspondencias croatas de la misma combinatoria sin que el significado cambie. Se trata de: si pues, pues fijate, pues vaya, pues nada, pues eso, pues bien y pues sí.

- Pues eso!, diga "nos hemos quedado sin casa", tal y como me lo cuenta a mí.

- Pa to / Pa tako / E tako, recite „ostali smo bez kuće“, onako kako meni govorite.

PIlAR. - Pues eso: tú tienes más confianza con él.

PILAR. - Pa (eto) to, više vjeruješ njemu.

Establecidos estos puntos, procederemos a indicar las diferentes traducciones de una específica combinatoria de las partículas de nuestro interés, siempre prestando atención tanto "al contexto como a los rasgos suprasegmentales que se superponen a dichas unidades (registro, entonación, pausas, acento, etc.)" (Martín Zorraquino y Portolés, 1998: 4145).

A la hora de presentar los equivalentes croatas y analizar dichas combinatorias, comenzaremos con pues ve. Para definir el concepto previamente introducido, al croata lo solemos traducir con $p a$, equivalente total de pues, combinado con el imperativo del verbo croata gledati 'mirar' - gledaj o gledajte-, siendo el primero la $2^{\mathrm{a}}$ p. sing. y el segundo la $2^{\mathrm{a}} \mathrm{p}$. pl. (en croata, el pronombre de cortesía). El primer elemento de la correspondencia croata puede variar, de ahí que en (2) sugiramos el conector $a$ 'pues' que encierra un cierto matiz de indiferencia:

(1) Pues ve, exactamente es una burla, pero no contra usted, en todo caso es usted el que se ha burlado de la muerte.

Pa gledajte, i jest ruganje, ali ne na Vaš račun, u svakom slučaju, Vi ste se narugali smrti.

(2) Pues ve, tu mamá le contagió su enfermedad desde que la tenía en la barriga y la niña ha ido a peor.

Pa gledaj / A gledaj, mama ju je zarazila još kad ju je nosila u trbuhu i djevojčici je sve gore i gore.

También, cabe señalar que, teniendo en cuenta el tratamiento de tú y Ud. en español, la traducción necesariamente cambiará. Por esa misma razón, en (1) optamos por gledajte ( $2^{\mathrm{a}} \mathrm{p}$. pl). En dichos ejemplos la técnica de traducción a la que hemos recurrido ha sido la de la variación en cuanto al estilo y el dialecto social (Olalla y Hurtado, 2014: 14)

A continuación, avanzaremos el análisis con pues fíjese. Como se verá más adelante, los tres ejemplos (3), (4) y (5) se traducen con pa 'pues', en combinación con el verbo croata gledati 'mirar' en imperativo (5) o con las expresiones fijas neće ići/moći [*no podrá ser] en (3) y nije tako ["o es así] en (4). Es notable la diferencia en la entonación de los ejemplos que siguen, igual que la semejanza entre (3) y (4) respecto al ritmo dentro del discurso. 
La comunicación entre el hablante y el interlocutor es más directa, más brusca, de ahí la decisión de enfatizar la traducción con un conjuntivo explicativo, en este caso $e$ 'ah'. Además de recurrir a la variación, aquí hemos empleado la técnica de traducción de amplificación (Olalla y Hurtado, 2014: 14) para introducir perifrasis explicativas croatas no formuladas en la combinatoria original del pues.

(3) Pues fijese que no, caballero. Podrá seguir después que le coloque la multa por la violación que acabe de cometer.

E pa neće ići/moći, gospodine. Možete nastaviti dalje nakon što vam napišem kaznu zbog prekršaja koji ste upravo počinili.

(4) -Pues fijese, padre, que no, y se lo digo con todo respeto.

- E pa nije tako, oče, i to Vam kažem s dužnim poštovanjem.

(5) Pues fijese, padre, que a mí me suena más dura la palabra estilo, probablemente porque conlleva un aire artificial, rebuscado.

A gledajte, oče, meni gore zvuči riječ stil, valjda jer je nekako umjetna...

Muy habituales en el habla cotidiana, pues hombre y pues venga muestran una alta frecuencia de uso, sobre todo en el ámbito oral. Por eso no debe extrañar que en (7), (8), (9) y (10) hayamos optado por utilizar las formas coloquiales, consideradas interjecciones en la lengua croata:

(6) ...hablando de controlar la angustia, y el dolor es una fuente de angustia y absolutamente clara, pues lo que hay que hacer es intentar realmente evitar cualquier tipo de dolor. No digo que la anestesia sea, pues hombre, pero la anestesia lo que hace es permitir trabajar cómodamente al profesional y también al paciente, de modo que hay que usarla, hay que usarla, sí.

... kad govorimo o kontroli tjeskobe, a bol je jasan izvor tjeskobe, ono što treba napraviti jest uistinu pokušati izbjeći svaku bol. Ne kažem da je anestezija rješenje, ali gle/vidi/daj, anestezija omogućava normalan rad i profesionalcu i pacijentu, tako da je definitivno treba koristiti.

(7) - Pues hombre, mucho gusto, me contesta, sin mostrar sorpresa alguna.

- Pa / E, drago mi je, odgovori mi, nimalo iznenađen.

(8) Muy bien, pues venga, pásame a Elena.

Dobro, 'ajde, daj mi Elenu.

(9) - Pues venga, pero con cuidado. Vamos, Valladar, deja ya de gruñir.

- 'Ajde/Polako/Idemo/'Ajmo, daj, ali oprezno. Daj, Valladar, prestani gunđati.

(10) —Pues venga, a comprar que han empezado las rebajas de enero!

- 'Ajmo/'Ajde/Idemo, u šoping, počela su zimska sniženja!

En cuanto a pues hombre, según la posición que ocupa dentro del discurso, la traducción obligatoriamente variará. En general, esta combinatoria del marcador discursivo pues "refuerza la imagen positiva del hablante, imprime un tono amistoso a la conversación y tiñe las relaciones entre los interlocutores de cierta familiaridad" (Martín Zorraquino y Portolés, 1998: 4173). Así observamos que en (7) lo traducimos con las partículas croatas pa 'pues' o $e$ 'ah', correspondencias que consideramos suficientes si tomamos en cuenta el contexto del discurso. En (6), debido a que pues hombre se encuentra en una posición intermedia, "está señalando un reinicio que aporta un comentario nuevo con respecto a lo dicho anteriormente" (Uribe Mallarino, 2005: 575). De ahí la decisión de traducirlo con el adversativo ali 
'pero', no porque se trate de dos argumentos completamente opuestos, sino para enfatizar y aclarar lo que viene en el segundo argumento, en combinación con las interjecciones croatas gle o daj. Cabe destacar que la técnica de traducción empleada en estos ejemplos ha sido, en primer lugar, la de la transposición, en otras palabras, se ha producido "un cambio en la estructura gramatical de una lengua a otra" (Duque Garcia, Trinidad González y Catrain, 1993: 139), en este caso del croata. Asimismo, aunque la posición dentro del discurso no varie, notamos un leve cambio en la entonación en (9) y (10), de ahí que en (9) optemos por suavizar la brusquedad de la correspondencia croata de pues con la interjección daj 'venga', recurriendo a otra técnica de traducción, la de la variación en cuanto al estilo, tono y el dialecto social-geográfico (Olalla y Hurtado, 2014: 14).

Como se verá a continuación, pues la verdad no presenta grandes problemas traductológicos ya que en la mayoría de los casos hemos optado por un adverbio en lugar de la verdad -zapravo 'realmente', iskreno 'sinceramente', najiskrenije [*lo más sinceramente]- reforzado con pa que corresponde a pues, empleando así la técnica de traducción de transposición (Olalla y Hurtado, 2014: 14). Sin embargo, en (11) hemos decidido emplear expresiones como pa da ti pravo kažem [*pues para decirte la verdad] y pa da ti budem iskren [*pues para serte sincero], visto que "la combinatoria ocupa la posición inicial y está seguida por una pausa, característica de los marcadores de tipo pragmático orientados al destinatario" (Uribe Mallarino, 2005: 575). Por tanto, nos deja más espacio para introducir la información a continuación:

(11) - Pues la verdad, no sé... Resulta todo muy vago, ¿no? ¿Qué te propones? - $\mathbf{P a} z a p r a v o / \mathbf{P a}$ iskreno / $\mathbf{P a}$ najiskrenije / $\mathbf{P a}$ da ti pravo kažem / $\mathbf{P a}$ da ti budem iskren, ne znam... Sve je nekako neodređeno, jel'da? Što si odlučio/naumio?

(12) "Lo mismo que llevo oyendo desde que comencé el viaje en Ferrol, y qué le iba a decir yo, pues la verdad: que ya me habia dado cuenta, y que no hay derecho." „Isto što slušam otkako sam krenuo na putovanje u Ferrol, a što da vam kažem, pa zapravo / pa iskreno / pa najiskrenije, da sam već shvatio da tu nema pravde."

En este caso la técnica de traducción empleada ha sido la de la amplificación (Olalla y Hurtado, 2014: 14).

Bueno pues es habitual en el ámbito oral y más bien, informal, en que "la conversación presenta una función interactiva orientada hacia el interlocutor" (Martín Zorraquino y Portolés, 1998: 4143). A este respecto, en (13) optamos por la interjección daj 'venga' (o incluso 'ajde više 'venga ya') con el fin de adaptarlo al registro croata ya que "su uso en exclamaciones encierra un matiz de enfado o protesta" (Calvi y Mapelli, 2004) lo que no ocurre en (14). En ese caso lo traducimos con una expresión dobro onda [*bueno pues] (equivalente total) que suaviza la brusquedad de una despedida:

(13) ILIADA: ¡Ey! ¡Bueno pues, Rodulfo! ¡Acaba de una vez la reprimenda! ILIADA: Ej! Daj / 'Ajde više, Rodulfo! Dosta više tog prigovaranja!

(14) Bueno pues, aquí me despido.

Dobro onda / Dakle, ja odoh/idem. 
En lo que atañe a la combinatoria pues mira (o variante mirá), hemos intentado conservar el matiz informal que dicha unidad conlleva en sí. Por tanto, cada correspondencia croata (traducida con el equivalente de miragle/vidi) la hemos enfatizado con pa 'pues'o a 'pues', y el resultado es un marcador conversacional ( $p a / a$ gle, $p a / a$ vidi). También, existe una posibilidad de traducir dicha combinatoria con una expresión muy empleada en el habla croata, (a) bože moj [*ah, Dios 'qué se le va a hacer'] (16), puesto que en la parte del discurso que introduce es notable el aire de resignación. En (17), pues mira es exclamativo y ocupa la posición inicial, lo que permite ofrecer varias correspondencias. Al final, recurriendo a la transposición y variación (Olalla y Hurtado, 2014: 14), hemos optado por el equivalente croata de 'imaginate' que en la lengua de partida manifiesta disgusto e indignación, reforzado con el conjuntivo $p a$, interjecciones coloquiales daj 'venga' o 'ajde 'venga', o hasta con la partícula ma 'pero'.

(15) Pues mirá, Horacio, si lo rechazás, te ocasionaremos un escándalo.

Pa vidi / Pa gle/ Dakle, ovako, Horacio, ako odbiješ, priredit ćemo ti skandal.

(16) Intentaremos convencerle de que la ventana no es para tirarse, es para tomar el aire; pero si no le convencemos, pues mira, qué le vamos a hacer.

Ali ako je ne uvjerimo, a vidi / a gle / (a) bože moj, što se može.

(17) ABELARDO. ¡Pues mira...! Después de tó lo que habremos bregao..., ahora que podemos disfrutar un poco, solo faltaba que nos quiten la pensión.

ABELARDO. Pa zamisli / Ma zamisli / 'Ajde zamisli/reci / Daj (samo) zamisli! Nakon tolike borbe, sad kad malo možemo uživati, samo nam nedostaje da nam uzmu penziju.

Con respecto a pues entonces, también característico del estilo informal, es obvia la desigualdad en la entonación en los siguientes ejemplos. Por tal motivo, en (18) optamos por una partícula con función especial en el texto, que en este caso sería onda 'entonces', añadiéndole equivalentes traductológicos de pues ( $p a$ o i) que "ayuda a solicitar una respuesta o conclusión al interlocutor" (Calvi y Mapelli, 2004). Pues entonces en (19) es exclamativo, lo que hemos logrado conservar en croata con onda 'entonces' y pa da ["pues sí], ampliando el primero con $p a$ 'pues' y el segundo con $e$ 'ah' que les dan ese toque explicativo, con un matiz de furia o molestia que podría concluir el discurso empezado. Incluso, como veremos en (19), empleando la técnica de traducción de variación (Olalla y Hurtado, 2014: 14), existe la posibilidad de combinar $p a$ ['pues] con la $2^{\text {a }} \mathrm{p}$. sing. del v. vidjeti 'ver' - pa vidiš ['ya ves]:

(18) Pues entonces, ¿a qué esperas para decirle que sí?

Pa onda / I onda, što čekaš? Odgovori mu da hoćeš.

(19) ¡Pues entonces! ¡Eso es lo que yo quería decirle! ¿Dónde está el problema?

(E) Pa vidiš! / Pa onda! (E) pa da! To sam mu i ja i želio reći! U čemu je problem?

Otra combinatoria del pues, la de así pues, combinación de dos adverbios contiguos que perdieron su significado conceptual, se caracteriza por ser la 
más frecuente en el presente corpus de investigación. Registrada mayoritariamente en el discurso escrito, cuyos equivalentes corresponden a en consecuencia y por lo tanto, esta combinatoria "vincula un primer miembro con otro que se presenta como su consecuencia" (Martín Zorraquino y Portolés, 1998: 4100) y se traduce con varios tipos de conectores, siempre dependiendo del contexto. En (20), teniendo en cuenta, en primer lugar, su valor conclusivo y consecutivo, luego la entonación y el contexto, la traducimos, empleando la técnica de amplificación (Olalla y Hurtado, 2014: 14), con la expresión znači da [*significa que]. Así pues en (21) es más bien explicativo que "relaciona y enfatiza lo dicho en el primer argumento introduciendo una conclusión argumentativa" (Calvi y Mapelli, 2004); de ahí las traducciones correspondientes: tako da 'así que', što bi značilo [*lo que significaria]:

(20) Se sentó con las piernas levemente separadas, sacó una revista de la bolsa y se puso a hojearla. Así pues, existía. Tenía que hablar con ella a toda costa...

Sjela je, lagano raširila noge, iz torbe izvadila časopis i počela ga listati. Znači da je postojala. Morao sam razgovarati s njom pod svaku cijenu.

(21) Soy hombre del Benemérito, y bien se sabe que un escándalo de estos podría dañar mi carrera política. Asi pues, vino el divorcio simple, si mayores estruendos.

Ja sam cijenjen čovjek i jasno je da bi mi takav skandal mogao uništiti političku karijeru.

Tako da / Što bi značilo da je razvod bio miran, bez velike buke.

\section{A MODO DE CONCLUSIÓN}

De acuerdo con el análisis traductológico, utilizando una serie de ejemplos provenientes del corpus del español, cabe destacar, en primer lugar, la escasa atención que se ha dedicado a los marcadores discursivos en la lingüistica croata (con excepción de Velčić, 1987), lo que aumenta las dificultades en la comprensión y la traducción del español al croata de unidades en cuestión, tomando en cuenta "las posibles discrepancias entre el significado de procesamiento en ambas lenguas" (Calvi y Mapelli, 2004). En segundo lugar, desde una perspectiva polifuncional, se ha comprobado que las funciones más frecuentes de las combinatorias del marcador discursivo pues son comentadora (lleva la atención hacia un elemento anterior añadiendo nuevos elementos), enfatizadora (matiza una información) y consecutiva (introduce una conclusión argumentativa), y que pueden encerrar diferentes matices, según la situación comunicativa. También, a lo largo del análisis, se ha confirmado que "en la mayoría de los casos el pues refuerza el sentido de la palabra que acompaña" (Uribe Mallarino, 2005: 566) y que forma parte de su combinatoria, igual que "se presenta como un comentario nuevo e informativamente valioso con respecto del discurso que lo procede" (Martín Zorraquino y Portolés, 1998: 4083).

Finalmente, si comparamos las correspondencias en las lengua de destino resumidas en la Tabla 3., a modo de resultados preliminares podemos concluir que la lengua de partida goza de marcadores más fijos y de significado universal, hasta en un vasto territorio como lo es el hispanófono, mientras que en la lengua de destino no se han lexicalizado clases de palabras 
tradicionales y estables con función del marcador de discurso, sino que los equivalentes se corresponden a variadas locuciones, expresiones, que difieren tanto en el registro como en el sentido diatópico, diafásico e idiolectal. En otras palabras, la diferencia entre los marcadores Pa zamisli / Ma zamisli / 'Ajde zamisli/reci / Daj (samo) zamisli, equivalente del español pues mira dependerá no tanto del contexto comunicativo sino de las preferencias del hablante, de la región en la que ha nacido, del idiolecto que cultiva. Por lo tanto, a la hora de describir lexicográficamente, en un diccionario bilingüe, los marcadores del discurso del español, el investigador deberá tener en cuenta un mayor número de ejemplos españoles, así como considerar no solamente las variedades diatópicas y diafásicas sino las preferencias idiolectales de los hablantes de una lengua tan pequeña como es el croata.

\section{REFERENCIAS}

ALCINA, J. \& BLECUA, J. M. (1975): Gramática española, Barcelona: Ariel, S. A.

BRIZ GÓMEZ, A. (1993a): "Los conectores pragmáticos en español coloquial (I): su papel argumentativo", Contextos, 11, 21/22, pp. $145-188$.

BRIZ GÓMEZ, A. (1993b): "Los conectores pragmáticos en español coloquial (II): su papel metadiscursivo", Español Actual, 59, pp. 39-56.

BRIZ GÓMEZ, A. (1994): "Hacia un análisis argumentativo de un texto coloquial. La incidencia de los conectores pragmáticos", Verba: Anuario galego de filoloxia, 21, pp. 369-395.

BRIZ GÓMEZ, A. (2001): El español coloquial en la conversación. Esbozo de pragmagramática, Barcelona: Ariel, S. A.

BRIZ GÓMEZ, A. e HIDALGO, A. (1998): "Conectores pragmáticos y estructura de la conversación", en MARTÍN ZORRAQUINO, Ma. A. y MONTOLÍO, E. (eds.), Marcadores discursivos: teoría y práctica, pp. 121-142.

BRIZ GÓMEZ, A. \& PONS, S. y PORTOLÉS, J. (coords.) (2008): Diccionario de partículas discursivas del español, Publicación electrónica:

http://www.dpde.es.

CALVI, M. V. \& MAPELLI, G. (2004): "Los marcadores bueno, pues, en fin, en los diccionarios de español e italiano", Artifara, 4, Publicación electrónica: http://www.artifara.com/rivista4/testi/marcadores.asp.

CASADO VELARDE, M. (1991): "Los operadores discursivos es decir, esto es, o sea y a saber en español actual: valores de lengua y funciones textuales", $L E A, 13$, pp. 87-116.

CASADO VELARDE, M. (1993): Introducción a la gramática del texto español, Madrid: Arco/Libros.

CASCÓN MARTÍN, E. (2006): Español coloquial. Rasgos, formas y fraseología de la lengua diaria, Madrid: Gráficas Glodami.

CUETO VALLVERDÚ, N. y LÓPEZ BOBO, M ${ }^{\mathrm{a}}$. (2003): La interjección. Semántica y Pragmática, Madrid: Arco/Libros.

DOMÍNGUEZ GARCÍA, N. (2016): "Bueno, pues, es que... en fin: ¿Qué marcadores discursivos enseñar?", Revista signos, versión On-line, 49, pp. 90.

DOMÍNGUEZ GARCÍA, N. \& DORTA LUIS, J. (2006): "La prosodia del marcador discursivo pues", Análisis del discurso: lengua, 
cultura, valores: Actas del I Congreso Internacional, 2, pp. 1269-1282.

DUQUE GARIAA, $\mathrm{M}^{\mathrm{a}}$. M., TRINIDAD GONZÁLEZ， M ${ }^{\mathrm{a}}$. \& CATRAIN, M. (1993): "Transposición y modulación en la traducción técnica", en RADERS, M. y SEVILLA, J. (eds.), III Encuentros complutenses en torno a la traducción, 2, pp. 137-151.

ESCANDELL VIDAL, $\mathrm{M}^{\mathrm{a}}$. (1996): Introducción a la pragmática, Barcelona: Ariel, S. A.

FUENTES RODRÍGUEZ, C. (1993): "Comportamiento discursivo de bueno, bien, pues bien", Estudios de Lingüistica (Universidad de Alicante), 9, pp. 205-221.

FUENTES RODRÍGUEZ, C. (2010): Diccionario de conectores y operadores, Madrid: Arco/Libros.

GILI GAYA, S. (1943): Curso superior de sintaxis española. Vox / Bibliograf.

GRAJALES ALZATE, R. (2011): "Funciones del marcador discursivo pues en el habla de Medellin, Colombia", Forma y Función, 24, 1, pp. 25-45.

GUEVARA, G. (2015): "Funciones del marcador discursivo pues: en un corpus oral", Revista Multidisciplinaria Dialógica, 12, pp. 294-323.

LA ROCCA, M. (2011): "Los marcadores del discurso en los manuales de Español/LE (19992010)", redELE, 21.

LAMÍQUIZ, V. (1994): El enunciado textual. Análisis lingüístico del discurso, Barcelona: Ariel Lingüistica.

LÓPEZ GARCÍA, A. (1994): Gramática del español I: La oración compuesta, Madrid: Arco/Libros.

LLOPIS-CARDONA, A. (2014): Aproximación funcional a los marcadores discursivos. Análisis $y$ aplicación lexicográfica, Frankfurt am Main, Berlin, Bern,
Bruxelles, New York, Oxford, Wien: Peter Lang Edition.

LLOPIS-CARDONA, A. (2014): "Las funciones pragmático-discursivas de en este sentido", Revista de Filología Española, 94, 1, pp. 127-150.

MARTÍ SÁNCHEZ, M. (2008): Los marcadores en Español/LE: conectores discursivos $y$ operadores pragmáticos, Madrid: Arco/ Libros.

MARTÍ SÁNCHEZ, M. (2011): "Los conectores discursivos (entre los otros marcadores discursivos y los otros conectores)", LINRED, 9, pp. 1-37.

MARTÍ SÁNCHEZ, M. (2013): Los marcadores discursivos. Para estudiantes y profesores. Español como lengua extranjera, Madrid: Edinumen.

MARTÍN ZORRAQUINO, Ma. A. (1992): "Partículas y modalidad", en HOLTUS, G. y SCHMITT, Ch. y METZELTIN, M. (eds.), Lexikon der romanistischen Linguistik, 6, 1. Tübingen: Max Niemeyer, pp. 110-124.

MARTÍN ZORRAQUINO, Ma. A. \& MONTOLÍO, E. (1998): Marcadores discursivos: teoría y práctica, Madrid: Arco/Libros.

MARTÍN ZORRAQUINO, Ma. A. \& PORTOLÉS LÁZARO, J. (1998): "Los marcadores del discurso", en BOSQUE, I. y DEMONTE, V. (dir.): Gramática descriptiva de la lengua española, Madrid: Espasa Calpe, S. A.

MARTÍNEZ, M. (1997): El paradigma emergente: hacia una nueva teoría de la racionalidad científica, México: Trillas, S. A.

OLALLA, C. \& HURTADO, A. (2014): "Estudio empírico de la traducción de los culturemas según el grado de adquisición de la competencia traductora. Un estudio exploratorio", Sendebar, 25, pp. 9-38. 
PONS, S. (1998a): Conexión y conectores. Estudio de su relación en el registro informal de la lengua, Cuadernos de Filología, Anexo 27, Valencia: Universitat de València.

PONS, S. (1998b): "Oye y mira o los límites de la conexión”, en Marcadores discursivos: teoria $y$ práctica, en MARTÍN ZORRAQUINO, Ma. A. y MONTOLÍO, E. (eds.), Marcadores discursivos: teoría y práctica, pp. 213-228.

PORTOLÉS, J. (1998): Marcadores del discurso, Barcelona: Ariel, S. A.

REAL ACADEMIA ESPAÑOLA. Corpes XXI - Corpus del Español del siglo XXI. Disponible en <http:/ / web.frl.es/COR-

$\mathrm{PES} /$ view/inicioExterno.view> [marzo 2016].

REAL ACADEMIA ESPAÑOLA: Banco de datos (CORDE) [en línea]. Corpus diacrónico del español. Disponible en <http://www.rae.es> [marzo 2016].

REAL ACADEMIA ESPAÑOLA: Banco de datos (CREA) [en linea]. Corpus de referencia del español actual. Disponible en <http://www.rae.es> [marzo 2016].

RE, A. (2010): "Conversación y marcadores en el aula de E/LE: los casos de bueno, pues y hombre", Trabajo Fin de Máster (Universidad de Salamanca).
REYES, G. (1994): La pragmática lingüistica. El estudio del uso del lenguaje, Barcelona: Montesinos Editor, S.L.

REYES, G. (2011): El abecé de la pragmática, Madrid: Arco/Libros.

SANTANA MARRERO, J. (2014): "Marcadores del discurso de Sevi1la", Cuadernos de La Alfal, 5, pp. 277-311.

SERRANO, M. J. (1997): "Marcadores discursivos en español: acerca de la verdad y pues", BFUCh, 36, pp. 265-286.

URIBE MALLARINO, Ma . (2005): “Conectores y contrastividad: el caso de pues", AISPI, 23, pp. 563-578.

URIBE MALLARINO, M ${ }^{\mathrm{a}}$. (2006): Los estudios sobre el conector pues, Milano: Led on line - Edizioni Universitaris di Lettere Economia Dritto.

VÁZQUEZ CARRANZA, A. (2013): "Responding and clarifying: an analysis of pues as a sequential marker in Mexican Spanish talk-in-interactions", Spanish in Context, 10, 2, pp. 284-309.

VÁZQUEZ CARRANZA, A. (2015): "Análisis de oye como marcador secuencial y de acción en la conversación", Estudios de Lingüística Aplicada, 61, pp. 73103.

VELČIĆ, M. (1987): Uvod u lingvistiku teksta, Zagreb: Školska knjiga. 